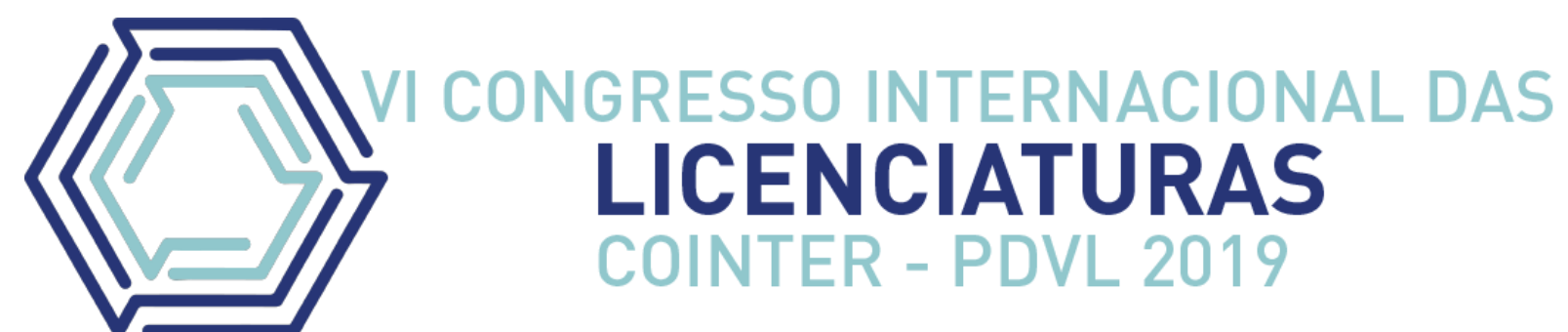

\title{
UMA BREVE CONSIDERAÇÃO ACERCA DA AVALIAÇÃO DA APRENDIZAGEM EM INSTITUIÇÕES DE EDUCAÇÃO BÁSICA NO MUNICÍPIO DE URUÇUÍ (PI)
}

\author{
BREVE CONSIDERACIÓN SOBRE LA EVALUACIÓN DEL APRENDIZAJE EN \\ INSTITUCIONES DE EDUCACIÓN BÁSICA EN LA CIUDAD DE URUÇUí (PI)
}

\section{A BRIEF CONSIDERATION ABOUT THE EVALUATION OF LEARNING IN BASIC EDUCATION INSTITUTIONS IN THE CITY OF URUÇUÍ (PI)}

\author{
Apresentação: Comunicação Oral \\ Isa Maria Antunes de Sousa ${ }^{1}$; Brunno Henryco Borges Alves²; José Ericson Lima dos Santos ${ }^{3}$ \\ Tyago Henrique Alves Saraiva Cipriano ${ }^{4}$; Ícaro Fillipe de Araújo Castro ${ }^{5}$ \\ DOI: https://doi.org/10.31692/2358-9728.VICOINTERPDVL.2019.0070
}

\begin{abstract}
Resumo
Muitos professores utilizam a avaliação somente como ferramenta punitiva e classificatória, sem se preocupar com a função de diagnóstico e aprendizado que deve estar associado a ela; é nessa perspectiva que as avaliações tradicionais vêm sendo bastante criticadas. O objetivo deste trabalho foi entender como a avaliação da aprendizagem é geralmente utilizada (ferramenta educativa ou classificatória) no ensino médio pelos professores de Biologia das escolas da rede pública Unidade Escolar Jose Patrício Franco e Instituto Federal de Educação Ciência e Tecnologia do Piauí - IFPI campus Uruçuí, do município de Uruçuí-PI. Desenvolveu-se a pesquisa a partir da aplicação de questionários semiestruturados formados por quatro questões abertas e seis fechadas, para quatro professores de Biologia. Os questionários buscavam informações referentes ao processo de aplicação de avaliação da aprendizagem, bem como esta é geralmente utilizada (ferramenta educativa ou classificatória) no ensino médio pelos professores. Observou-se que apesar de uma grande parcela dos professores terem encontrado lacunas em se tratando de sua formação acadêmica para o exercício da docência, bem como a falta de diálogos acerca da avaliação da aprendizagem, os mesmos se mostram cientes do que dizem os dispositivos legais que regulamentam os

\footnotetext{
${ }^{1}$ Licenciatura em Ciências Biológicas, Instituto Federal de Educação Ciência e Tecnologia do Piauí - IFPI campus Uruçuí, e-mail: isaantunes1999@gmail.com

${ }^{2}$ Licenciatura em Ciências Biológicas, Instituto Federal de Educação Ciência e Tecnologia do Piauí - IFPI campus Uruçuí, e-mail: brnnhnrc@gmail.com

${ }^{3}$ Licenciatura em Ciências Biológicas, Instituto Federal de Educação Ciência e Tecnologia do Piauí - IFPI campus Uruçuí, e-mail: ericson.alvo@gmail.com

${ }^{4}$ Licenciatura em Ciências Biológicas, Instituto Federal de Educação Ciência e Tecnologia do Piauí - IFPI campus Uruçuí, e-mail: tyagohenrique98@gmail.com

${ }^{5}$ Professor Mestre, Instituto Federal de Educação Ciência e Tecnologia do Piauí - IFPI campus Uruçuí, e-mail: icaro.castro@ifpi.edu.br
} 
critérios para verificação do rendimento escolar, assim como o desenvolvimento de avaliações alternativas com seus alunos.

Palavras-chave: diagnóstico, ferramenta, classificatória, lacunas.

\title{
Resumen
}

Muchos maestros usan la evaluación solo como una herramienta punitiva y de clasificación, sin preocuparse por la función de diagnóstico y aprendizaje que debería estar asociada a ella; Es desde esta perspectiva que las evaluaciones tradicionales han sido ampliamente criticadas. El objetivo de este trabajo fue comprender cómo la evaluación del aprendizaje es generalmente utilizada (herramienta educativa o de clasificación) en la escuela secundaria por los maestros de biología de las escuelas públicas Unidade Escolar Jose Patrício Franco y Instituto Federal de Educação Ciência e Tecnologia do Piauí - IFPI campus Uruçuí- PI. La investigación se desarrolló a partir de la aplicación de cuestionarios semiestructurados formados por cuatro preguntas abiertas y seis cerradas, para cuatro profesores de biología. Los cuestionarios buscaron información sobre el proceso de aplicación de la evaluación del aprendizaje, así como su uso general (herramienta educativa o de clasificación) en la escuela secundaria por parte de los maestros. Aunque una gran parte de los docentes encontraron lagunas en su capacitación académica para la enseñanza, así como la falta de diálogos sobre la evaluación del aprendizaje, son conscientes de lo que dicen las disposiciones legales. que regulan los criterios para verificar el rendimiento escolar, así como el desarrollo de evaluaciones alternativas con sus estudiantes.

Palabras Clave: diagnóstico, ferramienta, clasificatorio, lagunas.

\begin{abstract}
Many teachers use assessment only as a punitive and classifying tool, without worrying about the diagnostic and learning function that should be associated with it; It is from this perspective that traditional evaluations have been widely criticized. The objective of this work was to understand how the assessment of learning is generally used (educational or classifying tool) in high school by teachers of Biology of public Unidade Escolar Jose Patrício Franco and Instituto Federal de Educação Ciência e Tecnologia do Piauí - IFPI campus Uruçuí, do município de Uruçuí-PI. The research was developed from the application of semi-structured questionnaires formed by four open and six closed questions, for four biology teachers. The questionnaires sought information regarding the application process of learning assessment, as well as it is generally used (educational or classifying tool) in high school by teachers. Although a large portion of the teachers found gaps in their academic training for teaching, as well as the lack of dialogues about the assessment of learning, they are aware of what the legal provisions say. that regulate the criteria for verifying school performance, as well as the development of alternative assessments with their students.
\end{abstract}

Keywords: diagnosis, tool, classificatory, gaps. 


\section{Introdução}

Entre as funções da Educação, o preparo para o exercício da cidadania deve ser destacado, considerando que as demais são alicerces para esta. Assim, acrescenta-se, aqui, o dever de formar um ser humano livre, responsável, autônomo e capacitado para interagir no meio social, bem como possuir posicionamentos esclarecidos e críticos relativamente às questões do mundo de hoje (BRITO; LOPES, 2014).

A Escola, agente através do qual a educação formal atua, busca condições favoráveis à igualdade social, através do processo de formação do conhecimento. Dispondo de um ambiente favorável à transmissão de conhecimentos científicos, assim como de uma aprendizagem real para aquele que a recebe (OLIVEIRA et al., 2013).

O professor tem papel fundamental no processo de construção do conhecimento, entretanto, o aluno não é apenas um mero aprendiz nesse processo, tendo em vista que a escola possui como uma das suas principais políticas o processo de ensino-aprendizagem, ou seja, o ensino somente será efetivo, quando houver aprendizagem. Lopes (2009), diz que só é possível acontecer uma educação adequada se houver uma interação entre as ideias no âmbito educacional, seguindo a perspectiva de que a valorização do conhecimento só ocorre quando os envolvidos pensam e agem em conjunto.

A relação professor-aluno pode ser afetada por diversos aspectos, dentre eles destacase a avaliação da aprendizagem. Muitos professores utilizam a avaliação somente como ferramenta punitiva e classificatória, sem se preocupar com a função de diagnóstico e aprendizado que deve estar associada a ela. Segundo Cruz e Ferreira (2016), esse tipo de

avaliação, conhecida como tradicional, ainda se encontra muito presente nas escolas e valoriza mais os resultados quantitativos do que os qualitativos. Contrapondo o que diz a Lei de Diretrizes e Bases da Educação Nacional (LDB), a qual afirma que a avaliação deve ser contínua, levando em consideração todo o desempenho do aluno, com ênfase nos aspectos qualitativos em relação aos quantitativos (BRASIL, 1996).

No ensino de Biologia, a maior parte das aulas é voltada para aplicação de conteúdos de cunho investigatório. Assim, é essencial que o professor elabore situações práticas que possibilitem aos alunos gerenciar investigações para o desenvolvimento de teorias 
(TEODORO; CAMPOS, 2016). Essa disciplina também é fortemente marcada pela avaliação tradicional, contudo, Vidotto et al. (2013), afirmam que avaliações alternativas deveriam ser frequentemente utilizadas nas disciplinas de ciência natural, como a Física, Química ou Biologia, para extrair do aluno à relação teoria e evidência na prática.

A presença do modelo tradicional ainda influencia fortemente escolas e docentes de Biologia, o que torna em muitos casos a avaliação escolar meramente mecânica e punitiva, em que o foco deixa de ser o aprendizado e passa a ser um método apenas de distribuição de notas e classificação dos alunos. Nesse sentido, justifica-se o desenvolvimento do presente estudo, objetivando entender como a avaliação da aprendizagem é geralmente utilizada (ferramenta educativa ou classificatória) no ensino médio pelos professores de Biologia nas escolas da rede pública de Uruçuí - PI.

\section{Fundamentação Teórica}

As concepções que permeiam a avaliação no contexto escolar vêm desde os primórdios. Conforme Luckesi (2003) explicita, a tradição dos exames escolares, que se tem conhecimento atualmente, na maioria das escolas, foi sistematizada nos séculos XVI e XVII, com as configurações da atividade pedagógica produzidas pelos padres jesuítas e por Comênio. Assim, reporta às práticas de avaliação em forma de exames e provas, usadas nos colégios católicos jesuíticas e em escolas protestantes.

Diante disso, ainda hoje, nas escolas brasileiras, seja de modalidade pública ou particular, há a prática dos exames escolares em vez de avaliação de aprendizagem. Luckesi (2003) afirma: historicamente, passamos a denominar a prática de acompanhamento da avaliação da aprendizagem do educando de "Avaliação da aprendizagem escolar", mas, na verdade, continuamos a praticar "exames". Deste modo, o autor denomina de Pedagogia do Exame tal prática que, segundo ele, ainda se faz presente nas escolas da atualidade.

Nessa perspectiva, a prática de avaliação dos processos de ensino e de aprendizagem ocorrem por meio das relações pedagógicas, nas quais o professor atua como o avaliador desse processo atribuindo significados à avaliação. Nesse sentido, Sordi (2001) afirma que "uma avaliação espelha um juízo de valor, uma dada concepção de mundo e de educação, e por isso vem impregnada de um olhar absolutamente intencional que revela quem é o educador quando interpreta os eventos da cena pedagógica”. Assim, a avaliação requer intencionalidade e 
atitudes dos sujeitos envolvidos.

Dias Sobrinho (2003) afirma que, nas primeiras décadas do século passado, avaliar se confundia com medir. Embora consideremos hoje importante distinguir avaliação e medida, naquele momento, esses termos se tomavam um pelo outro. A avaliação era eminentemente técnica, consistindo basicamente em testes de verificação, mensuração e quantificação de resultados". Assim, a ideia de avaliar, não só para medir mudanças comportamentais, mas também a aprendizagem.

Segundo Caldeira (2000) a avaliação escolar é um meio e não um fim em si mesma; está delimitada por uma determinada teoria e por uma determinada prática pedagógica. Ela não ocorre num vazio conceitual, mas está dimensionada por um modelo teórico de sociedade, de homem, de educação e, consequentemente, de ensino e de aprendizagem, expresso na teoria e na prática pedagógica. Nessa perspectiva, a avaliação atua como uma ferramenta que é determinada pelas concepções que baseiam as propostas de ensino.

Álvarez Méndez (2002) afirma que o conhecimento deve ser o referente teórico que dá sentido global ao processo de realizar uma avaliação, podendo diferir segundo a percepção teórica que guia a avaliação. Aqui está o sentido e o significado da avaliação e, como substrato, o da educação. Assim, o ato de avaliar é uma atividade essencial da ação educativa, pois está ligado completamente ao conhecimento, visto que, avaliação da aprendizagem impulsiona o processo de construção do conhecimento.

Uma das percepções mais tradicionais sobre a avaliação na escola refere-se à possibilidade de classificar o desempenho do aluno por meio da mesma. Nessa perspectiva, Perrenoud (1999) declara que, a avaliação é tradicionalmente associada, na escola, à criação de hierarquias de excelência. Os alunos são comparados e depois classificados em virtude de uma norma de excelência, definida em absoluto ou encarnada pelo professor e pelos melhores alunos. Dessa forma, se dá a função da avaliação dentro do contexto escolar como classificatória. Para Esteban (2004), a avaliação classificatória não é somente um elemento justificador da inclusão/exclusão, ela está constituída pela lógica excludente dominante em nossa sociedade. Deste modo, à classificação dos alunos em uma escala caminha para causas excludentes.

O tradicional modelo de avaliação da aprendizagem escolar define a classificação de alunos como a principal função do ato de avaliar. Como aponta Luckesi (1984), a avaliação, na perspectiva do modelo tradicional, pode tornar-se um instrumento autoritário e frenador do desenvolvimento, possibilitando a uns o acesso ao saber e, a outros, a estagnação e até a evasão 
escolar. Neste sentido, a avaliação visa a classificar o indivíduo, segundo um padrão determinado.

Para Luckesi (1984), o modelo tradicional da avaliação escolar assume, como principal função do ato de avaliar, a classificação e não o diagnóstico. Nesse sentido, a avaliação classificatória pode tornar-se um instrumento punitivo e ceifador do processo de desenvolvimento escolar para muitos alunos.

Leite e Tassoni (2002) apontam que, a avaliação torna-se profundamente aversiva quando o aluno discrimina que as consequências do processo podem ser direcionadas contra ele próprio. De fato, o modelo de avaliação seguido pela grande maioria das escolas tem contribuído com os altos índices de fracasso escolar.

De acordo com Maciel (2003), se, de um lado, a escola usa a avaliação como instrumento de poder e de controle do aluno, por outro, o aluno acaba desenvolvendo estratégias de sobrevivência e criando um 'contra-poder' estabelecendo uma relação utilitarista com o saber e com o outro. Deste modo, a relação professor-aluno torna-se, muitas vezes, desamiga, pela relação de poder que se instaura na mesma.

Ao abordar as percepções pedagógicas que transpõem a avaliação no contexto escolar, constata-se que, inicialmente, o ato de avaliar e de examinar se assemelham. Esteban (2004) declara, embora muito criticada, a avaliação do desempenho escolar, como resultado do exame que o professor ou professora realiza sobre o aluno ou aluna, ainda é predominante. Dessa forma, faz necessário que revejam e se alterarem a,0s práticas de avaliação adotadas tradicionalmente pela maioria das escolas.

Observa-se também que avaliar tem-se confundido com medir a quantidade de conhecimentos adquiridos pelos alunos. Nesse sentido, Gatti (2003) afirma: é preciso ter presente, também, que medir é diferente de avaliar. Mas, a partir das medidas, para termos uma avaliação é preciso que se construa o significado dessas grandezas em relação ao que está sendo analisado quando considerado como um todo. Por isso o processo de avaliação deve considerar e envolver os valores sociais.

Vygotsky (1984), por sua vez, introduz um conceito fundamental para a aprendizagem e para o desenvolvimento: a mediação. É a partir de um intenso processo de interação com o meio social, através da mediação feita pelo outro, que se dá a apropriação dos bens culturais. Esse complexo processo resulta no desenvolvimento. Assim, o processo de avaliação da aprendizagem deve ser direcionado para o método de transformação social e para o crescimento 
e desenvolvimento integral dos alunos.

\section{Metodologia}

De acordo com Cardoso (2013), o presente estudo trata-se de uma pesquisa qualitativa, tendo em vista que esta buscou abordar em seus resultados a realidade vivenciada por cada aluno ou pelo grupo como um todo. O mesmo foi desenvolvido no município de Uruçuí-PI, onde foram aplicados questionários semiestruturados formados por quatro questões abertas e seis fechadas, para quatro professores de Biologia de duas escolas públicas do município de Uruçuí-PI.

Para esta pesquisa, ocorreu inicialmente uma visita as escolas participantes do estudo e solicitou-se a direção das escolas permissão para execução desse trabalho. Após essa concessão, professores de biologia das referidas escolas foram convidados para participar e esclarecidos da pesquisa por meio de um Termo de Consentimento Livre e Esclarecido (TCLE) que resguardava o anonimato dos participantes bem como os assegurava de a qualquer momento que desejar, não participar da pesquisa.

Para alcançar os objetivos desse trabalho, optou-se pelo questionário, por ser uma ferramenta, segundo Parasuraman (1991), um conjunto de questões, feito para gerar os dados necessários para se atingir os objetivos do projeto. Assim, o questionário é essencial na pesquisa científica, especialmente nas ciências sociais.

O questionário utilizado continha 11 perguntas, sendo: 3 abertas e 8 fechadas. Os questionários buscavam informações referentes ao processo de aplicação de avaliação da aprendizagem, bem sua forma de utilização (ferramenta educativa ou classificatória) no ensino médio pelos professores e foi confeccionado a partir de Rodrigues et al. (2016), Lemos e Sá (2013) e Camargo (2010). Os dados obtidos pelas respostas dos docentes foram trabalhados na plataforma Excel, a qual proporcionou a elaboração de gráficos e tabelas para representar as variáveis estudadas durante a pesquisa.

\section{Resultados e Discussão}

Foram entrevistados 4 professores de duas instituições de Ensino, sendo um homem e três mulheres com idade entre 28 e 36 anos. De acordo a Figura 1, todos os entrevistados 
apresentaram formação em nível de pós-graduação: 2 especialistas, 1 mestre e 1 doutor, em área específica ou docência do ensino superior. Para Jung (2007), a formação continuada bem como cursos de especialização ou programas de aperfeiçoamento do magistério podem proporcionar aos docentes um sentimento de curiosidade, o qual direciona para busca de compreensão da rotina do ambiente de trabalho, desenvolvendo novas metodologias em sua prática docente.

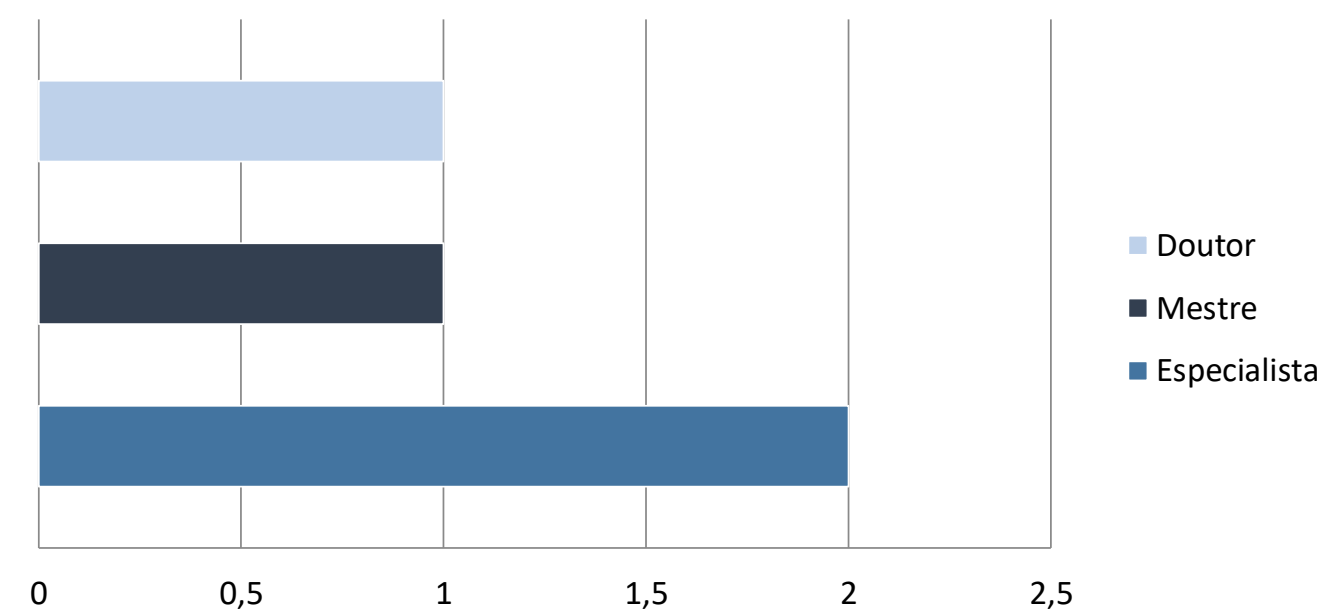

Figura 1: Nível de Pós-Graduação dos docentes de Biologia de Escolas Públicas do ensino médio do município de Uruçuí (PI)

Fonte: Arquivo pessoal.

Conforme se observa na Tabela 1, 50\% dos professores afirmaram que sua formação acadêmica os preparou bem para o exercício da docência. Segundo Rodrigues et al. (2018), em estudos sobre o processo de formação e atuação do professor, constataram que a participação em programas de iniciação à docência, bem como formas alternativas que favoreçam o preparo para o mercado de trabalho são aspectos positivos a serem trabalhados que irão contribuir para sua formação, e, consequentemente, proporcionar um conhecimento direto do ambiente de trabalho do professor. O que não é comtemplado por uma parcela considerável dos professores entrevistados, tendo em vista que $50 \%$ destes afirmaram que sua formação acadêmica não os preparou bem para o exercício da docência.

Tabela 1: Percepção dos professores de Biologia de Escolas Públicas do ensino médio do município de UruçuíPI sobre sua formação acadêmica.

Item Sim Não


Sua formação acadêmica o preparou bem para o exercício da docência? $\quad 50 \% \quad 50 \%$

No seu curso de graduação, o tema avaliação do ensino foi debatido em sala de aula?

$50 \% \quad 50 \%$

Barbosa (2012) afirmou que se fazem necessários mais diálogos, questionamentos e reflexões acerca do processo de avaliação de aprendizagem e sua importância para o exercício da docência nos cursos de formação de professores. Quando questionados sobre esses fatores, $50 \%$ dos entrevistados afirmaram que debates envolvendo o assunto foram desenvolvidos em sala de aula durante sua formação em nível e graduação (tabela 1). Em turmas de futuros professores a prática pedagógica, em se tratando da avaliação da aprendizagem, oportuniza aos estudantes debates sobre a educação, bem como o ato de avaliar a aprendizagem do outro (BARBOSA, 2012). No entanto, isso não foi contemplado nos cursos de graduação de 50\% dos professores entrevistados, que afirmaram que a avaliação da aprendizagem não ocupou destaque nas discussões em sala de aula.

A Lei de Diretrizes e Bases da Educação Nacional em seu inciso V do artigo 24 diz que a verificação do rendimento escolar deverá observar os seguintes critérios:
a) avaliação contínua e cumulativa do desempenho do aluno, com prevalência dos aspectos qualitativos sobre os quantitativos e dos resultados ao longo do período sobre os de eventuais provas finais;
b) possibilidade de aceleração de estudos para alunos com atraso escolar;
c) possibilidade de avanço nos cursos e nas séries mediante verificação do aprendizado;
d) aproveitamento de estudos concluídos com êxito;
e) obrigatoriedade de estudos de recuperação, de preferência paralelos ao período letivo, para os casos de baixo rendimento escolar, a serem disciplinados pelas instituições de ensino em seus regimentos; (BRASIL, 1996, p. 07).

Apesar da atenção expressa na LDB/96 para a avaliação de aprendizagem, Barcelos (2015) afirma que no Brasil os baixos índices de conclusão e elevados índices de evasão das escolas de Rede Pública, devem-se à ineficiência da administração pública escolar, falta de recursos financeiros, administrativos, e, principalmente, às más estratégias de avaliação de aprendizagem. Entretanto, quando questionados sobre a eficiência de suas práticas avaliativas, todos os docentes entrevistados afirmaram dispor de um método eficaz para avaliação dos discentes (Tabela 2), demonstrando consonância com o dispositivo legal que a regulamenta. 
Tabela 2: Concepção de professores de Biologia de Escolas Públicas do ensino médio do município de UruçuíPI acerca do processo de avaliação de aprendizagem.

\begin{tabular}{lcc}
\hline \multicolumn{1}{c}{ Item } & Sim & Não \\
\hline Você conhece o que a LDB fala sobre a avaliação? & $75 \%$ & $25 \%$ \\
\hline Você julga sua prática avaliativa eficaz? & $100 \%$ & $0 \%$ \\
\hline Você conhece a diferença entre avaliação qualitativa e quantitativa? & $100 \%$ & $0 \%$ \\
\hline $\begin{array}{l}\text { Você conhece os procedimentos/estratégias avaliativos como } \\
\text { observação e registro? }\end{array}$ & $100 \%$ & $0 \%$ \\
\hline Você utiliza esses métodos no dia-a-dia? & $75 \%$ & $25 \%$ \\
\hline
\end{tabular}

Em se tratando das concepções dos professores sobre o processo de avaliação, de acordo com Weber (2007), as avaliações de aprendizagem devem estar vinculadas às atividades didáticas, sendo estas participantes do processo de ensino e aprendizagem. Para Castanheira e Ceroni (2008), no mundo contemporâneo, a avaliação de aprendizagem é utilizada para selecionar, aprovar ou capacitar trabalhadores; contudo, quando associada à educação, esta deveria ser vista como prática pedagógica, no entanto, também são utilizadas apenas para selecionar. A definição de avaliação de aprendizagem é perceptível na fala de um dos entrevistados que diz: "Avaliação é uma etapa do processo de ensino aprendizagem que deve estar presente cotidianamente em sala de aula; sua função é obter um diagnóstico do aluno, considerando seus avanços. É também uma ação que exige do professor uma reflexão sobre sua prática pedagógica”. (Sr. A., 30 anos).

As avaliações tradicionais vêm sendo bastante criticadas principalmente por serem utilizadas como ferramenta punitiva aos alunos. É nessa perspectiva que Depresbiteris e Tavares (2017), dizem que meios alternativos de avaliação podem ser uma nova possibilidade para o professor. Dentre esses meios alternativos mencionados pelos autores, destacam-se nas respostas dos entrevistados: debates, seminários, relatórios e participação nas aulas. Contudo, Castro (2016) afirma que esses meios alternativos para o processo de avaliação da aprendizagem não dependem apenas da disposição do professor, mas também de qual postura a escola assume diante deste contexto. O que também é percebido em relatos dos entrevistados: "A instituição em que trabalho incentiva os dois tipos de avaliação, (qualitativa e quantitativa), porém, nas instituições privadas, o que conta mesmo são as avaliações quantitativas”. (Sr. O., 33 anos). 
Todos os professores entrevistados afirmaram conhecer o significado da avaliação qualitativa, bem como os procedimentos para sua execução. Por isso a colocam em prática na sua atuação docente. A avaliação qualitativa tem por definição a observação de tudo o que é desenvolvido em sala, levando em consideração se estão aprendendo, como estão aprendendo e quais suas principais dificuldades (ARAÚJO; MACADO, 2009).

\section{Conclusões}

A literatura atual apresenta muitas críticas sobre o processo de avaliação posto em prática por docentes para verificação do rendimento dos alunos, o qual não tem contemplado todas as dimensões do discente, deixando predominar os aspectos quantitativos sobre os qualitativos, contrariando o que determina a Lei de Diretrizes e Bases da Educação Nacional.

Os professores participantes da pesquisa mostraram-se cientes do que dizem os dispositivos legais que regulamentam os critérios para verificação do rendimento escolar, apesar de uma parcela considerável destes terem encontrado lacunas em sua formação para o exercício da docência quanto ao assunto abordado.

O processo de avaliação da aprendizagem do aluno não depende apenas do professor, mas também de quais atitudes são tomadas pela escola em se tratando deste procedimento. Entretanto, resta claro que avaliações alternativas estão sendo desenvolvida pelos professores em análise por meio de debates, seminários, relatórios e participação dos alunos nas aulas.

\section{Referências}

ÁLVAREZ MÉNDEZ, Juan M. Avaliar para conhecer: examinar para excluir. Porto Alegre: Artmed, 2002.

ARAÚJO, R. M. B.; MACADO, S. Os desafios da avaliação da aprendizagem, na prática do curso de Enfermagem, no Centro Universitário Metodista. Revista @ mbienteeducação, São Paulo, v. 2, n.1, p. 103-112, jan./jul. 2009. Disponível em: < http://publicacoes.unicid.edu.br/index.php/ambienteeducacao/article/view/564/532> Acesso em 01 junh. 2018.

BARBOSA, F. R. P. Avaliação da aprendizagem na formação de professores: estão os futuros professores preparados para avaliar?. IX ANPED SUL. 2012. Disponível em: < http://www.ucs.br/etc/conferencias/index.php/anpedsul/9anpedsul/paper/viewFile/2082/587> Acesso em 12 mai. 2018. 
BARCELOS, L. C. M. Avaliação da aprendizagem escolar. 2015. Pará de Minas. Disponível

em: <http://fapam.web797.kinghost.net/admin/monografiasnupe/arquivos/18072016193145Leda_ Cristina.pdf $>$ Acesso em 13 mai. 2018.

BRITO, A. C. A. G.; LOPES, M. E. O papel da educação escolar para o exercício da cidadania. PRIMUS VITAM. $\mathrm{N}^{\mathrm{o}} 7-2^{\circ}$. 2014. Disponível em: < http://mackenzie.br/fileadmin/Graduacao/CCH/primus_vitam/primus_7/azenath.pdf> Acesso em 20 abr. 2018.

CALDEIRA, Anna M. Salgueiro. Avaliação e processo de ensino-aprendizagem. Presença Pedagógica, Belo Horizonte, v. 3, p. 53-61, set./out.

1997.

CAMARGO, W. F. Avaliação da aprendizagem no ensino fundamental. Universidade Estadual de Londrina. 2010. Disponível em: http://www.uel.br/ceca/pedagogia/pages/arquivos/WANESSA\%20FEDRIGO.PDF> Acesso em 24 abr. 2018.

CARDOSO, F. S. O uso de atividades práticas no ensino de ciências: na busca de melhores resultados no processo ensino aprendizagem. 2013. 56f. Monografia do Curso de Graduação em Ciências Biológicas, do Centro Universitário UNIVATES. Rio Grande do Sul, Lajeado, 2013.

CASTANHEIRA, A. M. P.; CERONI, M. R. Formação Docente e a Nova Visão da Avaliação Educacional. Estudos em Avaliação Educacional, v. 19, n. 39, jan./abr. 2008. Disponível em: < http://www.fcc.org.br/pesquisa/publicacoes/eae/arquivos/1421/1421.pdf> Acesso em 13 mai. 2018.

CASTRO, G. R. Educação profissional em saúde: um estudo sobre a avaliação da aprendizagem na escola de formação técnica em saúde enfermeira izabel dos santos (RJ). Fundação Oswaldo Cruz Escola Politécnica De Saúde Joaquim Venâncio. 2016. Disponível em:

https://www.arca.fiocruz.br/bitstream/icict/15880/2/Gracinete_Castro_EPSJV_Mestrado_201 6.pdf> Acesso em 01 junh. 2018.

DEPRESBITERIS, L.; TAVARES, M. R. Diversificar é preciso... Instrumentos e técnicas da avaliação de aprendizagem. 2017. Disponível em: < https://books.google.com.br/books?hl=pt$\mathrm{BR} \& \mathrm{lr}=\& \mathrm{id}=\mathrm{VOg} \_$DwAAQBAJ\&oi=fnd\&pg $=\mathrm{PT} 2 \& \mathrm{dq}=\mathrm{m} \% \mathrm{C} 3 \%$ A9todos + para + avalia $\% \mathrm{C} 3$ $\% \mathrm{~A} 7 \% \mathrm{C} 3 \% \mathrm{~A} 3 \mathrm{o}+\mathrm{de}+$ aprendizagem\&ots $=65 \mathrm{fwFVTj} 12 \&$ sig $=\mathrm{fJ} 6 \mathrm{CI}$ zhPHby4SqfLKpSWEvV $\mathrm{JkQ} \# \mathrm{v}=$ onepage \&q=m\%C3\%A9todos\%20para\%20avalia\%C3\%A7\%C3\%A3o\%20de\%20apr endizagem \&f=false> Acesso em 01 junho. 2018.

DIAS SOBRINHO, José. Avaliação: políticas e reformas da Educação Superior. São Paulo: Cortez, 2003.

ESTEBAN, Maria Tereza. Pedagogia de Projetos: entrelaçando o ensinar, o aprender e o avaliar à democratização do cotidiano escolar. In: SILVA, J. F.; HOFFMANN, J.; 
ESTEBAN, M. T. (orgs.) Práticas avaliativas e aprendizagens significativas: em diferentes áreas do currículo. 3.ed. Porto Alegre: Mediação, 2004. p. 81-92.

GATTI, B. A. O Professor e a avaliação em sala de aula. Estudos em Avaliação Educacional, n. 27, p. 97-113, jan./jun. 2003.

JUNG, K. M. A pesquisa na formação do professor. 2007. Disponível em: < http://euler.mat.ufrgs.br/ vclotilde/disciplinas/pesquisa/texto_Jung.pdf> Acesso em 01 junh. 2018.

LEITE, S. A. S.; TASSONI, E. C. M. A afetividade em sala de aula: as condições de ensino e a mediação do professor. In: AZZI, R. G.; SADALLA, A. M. F. A. (Org.). Psicologia e formação docente: desafios e conversas. São Paulo: Casa do Psicólogo, 2002.

LEMOS, P. S.; SÁ, L. P. A AVALIAÇÃO DA APRENDIZAGEM NA CONCEPÇÃO DE PROFESSORES DE QUÍMICA DO ENSINO MÉDIO. Revista Ensaio, Belo Horizonte. v.15. n. 03. p. 53-71. 2013. Disponível em: < http://www.scielo.br/pdf/epec/v15n3/19832117-epec-15-03-00053.pdf> Acesso em 24 abr. 2018.

LOPES, R. C. S. A relação professor aluno e o processo ensino aprendizagem. 2009. Disponível em: < http://www.diaadiaeducacao.pr.gov.br/portals/pde/arquivos/1534-8.pdf> Acesso em 21 abr. 2018.

LUCKESI, C. C. Avaliação educacional escolar: para além do autoritarismo. Tecnologia Educacional, Rio de Janeiro, n. 61, 1984.

LUCKESI, Cipriano C. Avaliação da aprendizagem na escola: reelaborando conceitos e recriando a prática. Salvador: Malabares Comunicação e Eventos, 2003.

MACIEL, D. M. A avaliação no processo ensino-aprendizagem de matemática no ensino médio: uma abordagem formativa sócio-cognitiva. 2003. Dissertação (Mestrado em Educação) - Faculdade de Educação, Universidade Estadual de Campinas, Campinas, SP, 2003.

OLIVEIRA, T.; VIANA, A. P. S.; BOVETO, L.; SARACHE, M. V. Escola, conhecimento e formação de pessoas: considerações históricas. Políticas Educativas, Porto Alegre, v. 6, n.2, p. 145-160, $2013-$ ISSN: 1982-3207. Disponível em: < http://seer.ufrgs.br/index.php/Poled/article/download/45662/28843> Acesso em 20 abr. 2018.

PARASURAMAN, A. Marketing research. 2. ed. Addison Wesley Publishing Company, 1991. 
PERRENOUD, Philippe. Avaliação: da excelência à regulação das aprendizagens - entre duas lógicas. Porto Alegre: Artes Médicas Sul, 1999.

RODRIGUES, D. B.; OLIVEIRA, D. P. C.; SILVA, G. A. A.; ROCHA, S. S. Avaliação da aprendizagem no ensino médio: as concepções dos professores de física sobre o uso da observação e dos registros para avaliar. Educação em Debate. 2016. Disponível em: <http://repositorio.ufc.br/bitstream/riufc/21269/1/2016_art_dbrodrigues.pdf> Acesso em 24 abr. 2018.

RODRIGUES, F. F. S.; ARAÚJO, E. M. S.; COCCO, D. D. A.; RODRIGUES, L. F. O processo de formação e atuação do professor na perspectiva dos bolsistas do programa institucional de bolsa de iniciação à docência - PIBID. Getec, v.7, n.16, p.34-42, 2018. Disponível em: <file://C:/Users/Tyago\%20Henrique/Downloads/1329-4846-1-PB.pdf> Acesso em 12 mai. 2018.

SORDI, Mara Regina L. de. Alternativas propositivas no campo da avaliação: por que não? In: CASTANHO, Sérgio; CASTANHO, Maria Eugênia (orgs.). Temas e textos em metodologia do Ensino Superior. Campinas, SP: Papirus, 2001.

TEODORO, N. C.; CAMPOS, L. M. L. O professor de biologia e dificuldades com os conteúdos de ensino. SBEnBio - $\mathrm{N}^{\circ} 9$ - 2016 Disponível em: < http://www.sbenbio.org.br/wordpress/wp-content/uploads/renbio-9/pdfs/2371.pdf> Acesso em 22 abr. 2018.

VIDOTTO, L. C.; LABURÚ, C. E.; BARROS, M. A.; Uma comparação entre avaliação tradicional e alternativa no ensino médio de física. 2013. Disponível em: < http://www2.unifap.br/rsmatos/files/2013/10/Vidotto1.pdf> Acesso em 22 abr. 2018.

VYGOTSKY, L. S. A formação social da mente. São Paulo: Martins Fontes, 1984.

WEBER, S. S. F. Avaliação da aprendizagem escolar: práticas em novas perspectivas. Universidade Federal de Santa Maria - RS. 2007. Disponível em: < https://repositorio.ufsm.br/bitstream/handle/1/6785/SONIAWEBER.pdf?sequence=1\&isAllo wed $=y>$ Acesso em 20 mai. 2018. 